\title{
Performance Comparison of Hybrid GA-PSO Based Tuned IMMs for Maneuver Target Tracking
}

\author{
Ravi Kumar Jatoth \\ Assistant Professor, Department of ECE, National Institute of Technology-Warangal, India \\ E-mail: ravikumar@nitw.ac.in \\ Dr. T. Kishore Kumar \\ Ass ociate Professor, Department of ECE, National Institute of Technology-Warangal, India \\ E-mail:kishoret@nitw.ac.in
}

\begin{abstract}
Target tracking is very important field of research as it has wider applications in defense as well as civilian applications. Kalman filter is generally used for such applications. When the process and measurements are non linear extensions of Kalman filters like Extended Kalman Filter, Unscented Kalman Filters are widely used. UKF can give estimations up to second order characteristics of random process. The target is maneuvering and switching among different models like constant velocity (CV), constant acceleration (CA) or constant turn (CT), Interactive Multiple Models (IMM) are employed. Implementation of IMM filters for any application is difficult because of initialization of Kalman filter i,e, tuning of filter has to be performed before applying to real time situations. It demands prior estimations of Noise covariance matrices which are left for engineering intuitions. This paper presents the nonlinear state estimation using IMM and tuning of the filter is done using bio-inspired algorithms like PSO GA and Hybrid GA-PSO.
\end{abstract}

Index Terms - Extended Kalman Filter, Unscented Kalman Filter, Interactive Multiple Models, Target Tracking, Tuning of filter, Hybrid GA-PSO Algorithm

\section{Introduction}

In many tracking applications Kalman Filter (KF) is used to estimate the velocity, position and acceleration of a maneuvering target from no isy radar measurements at high data rates. Bearings only tracking is attracted many researches in these days due to its practical military and civilian applications [1-2]. When the process is to be estimated and measurement model is nonlinear, EKF is used in which, the process is approximated to first order term of the Taylor's expansion for calculating the mean and covariance of the random process [3]. This linearization however poses some problems e.g. it can produce highly unstable filters if the assumptions of local linearity is violated. In this paper we simulate UKF (estimator) which generalizes sophisticatedly to nonlinear systems without the linearization steps required by the EKF.

The UKF uses deterministic sampling approach [4]. Approximating a Gaussian distribution is easier than approximating a nonlinear transformation so state distribution is Approximated by a Gaussian random vector. Whenever the target is taking multiple switching among different models IMM are generally used. These EKF and UKF are used in parallel in interactive multiple models. The Kalman filter demands priori information about the noise covariances from the user [5]. In itial process and measurement noise covariances play an important role in convergence of the filter. If the noise covariances are not chosen properly it may leads towards degradation of the filter performance [6]. A few techniques for determining the process and measurement noise covariances for various applications have been discussed in the literature [7], [8] and widely used tuning method is least squares approach.

The Standard Genetic Algorithm (SGA) is inspired by Charles Darwin's evolutionary theory of evolution. Typically Genetic Algorithm maintains a population of candidate solutions for problem at hand and makes it evolve by iteratively applying a set of stochastic operations [9]. Particle Swarm Optimization (PSO) is population based stochastic optimization technique inspired by social behaviour of bird flocking or fish schooling in searching for food [10]. PSO exploits a population of individuals to probe promising regions of the search space. In the context, the population is called a swarm and the individuals are called particles. Each particle moves with an adaptable velocity within the search space, and retains in its memory the best position it ever encountered. In the global variant of PSO the best position ever attained by all individuals of swarms is communicated to all the particles. PSO and GA are population based heuristic search technique which can be used to solve the optimization problems modelled on 
the concept of Evolutionary Approach. In standard PSO, the non-oscillatory route can quickly cause a particle to stagnate and also it may prematurely converge on suboptimal solutions that are not even guaranteed to be local optimum. So A Hybrid GA-PSO algorithm is proposed [11].

This paper implements GA, PSO and GA-PSO based tuning of IMM-EKF and IMM-UKF, in which process noise and measurement covariances are tuned based on biologically inspired evolutionary computing tools.

\section{Problem Statement}

In this paper target tracking environment is taken as shown in "fig.1".

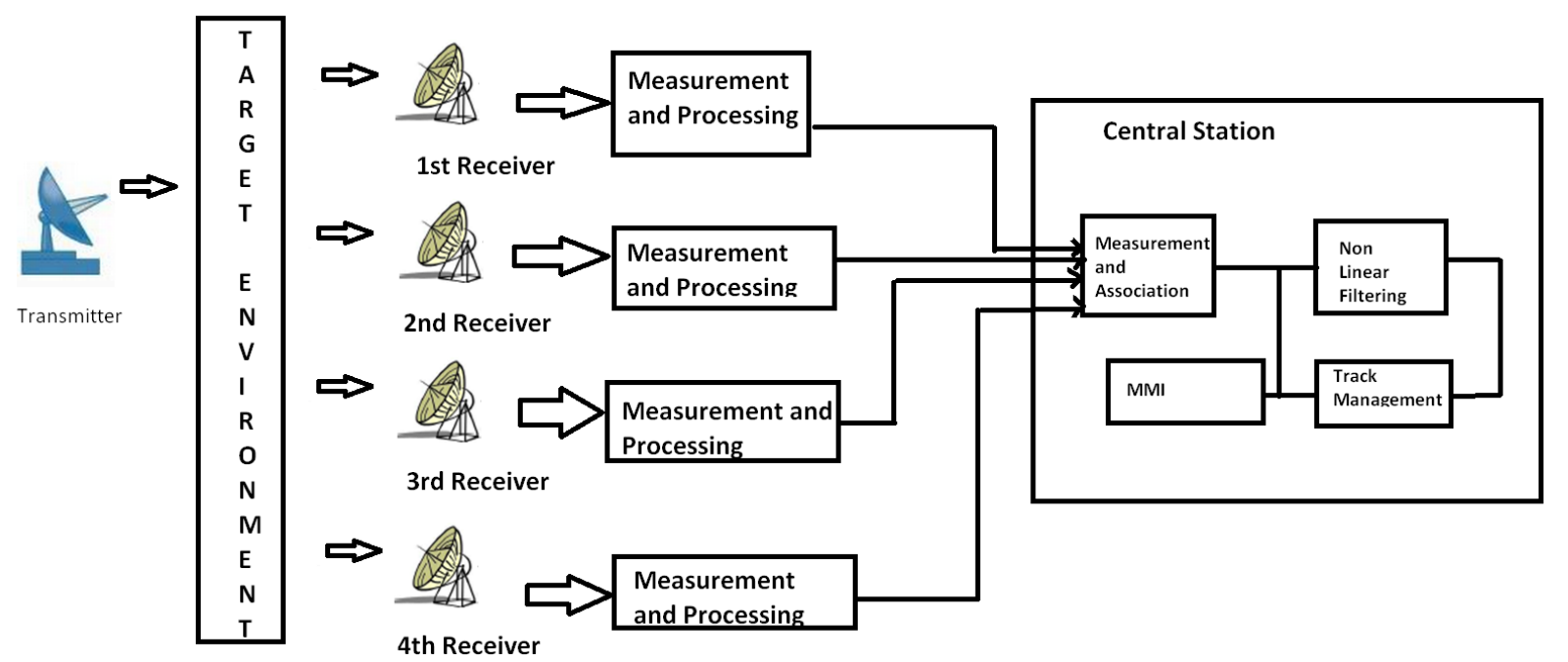

Fig. 1: Multiple sensor Tracking

Multisensor target tracking is finding many applications these days because of its advantages like accurate target tracking and cheaper in cost.

Measurement processing generally includes a form of thresholding (measurement detection) process. Information loss during the thresholding has to be taken care and in very low SNR scenarios, thresholding might not be used, which leads to Track before Detect algorithms with high computation cost. Detections originate not only from targets being tracked, but also from thermal noise as well as from various objects such as terrain; clouds and these unwanted measurements are usually termed clutter. Target trackers (TT) are widely used in air defense, ground target tracking, and missile defense. Target tracking have two portions: Data association algorithm section and estimation and prediction section. Data association is the process to match a measurement to a landmark .Gating is a technique for eliminating unlikely measurement-totrack pairings and the purpose of gating is to reduce computational expense by eliminating from consideration measurements which are far from the predicted measurement location [12]. Data association algorithms deal with situations where there are measurements of uncertain origin

We want to track the aircraft position by using sensors in presence of process noise and measurement noise. The measurements are in polar coordinates (bearing $\theta$ ) as we are using sensors (RADAR), which measure only the bearings (or angles) with respect positions of the sensors. Solving this problem is important, because more general multiple target tracking (MTT) problems can be partitioned to sub problems, in which single target is tracked separately. Basic problem is to estimate the target kinematic state (position and velocity) from noise corrupted measurements. Since the output of the filtering algorithm is required to be Cartesian position and velocity, the target Kine matic state can be described by the state vectordefined in discrete time as

$$
x_{k}=\left[x_{k}, y_{k}, v_{x_{k}}, v_{y_{k}}\right]^{T}
$$

Where $\mathrm{T}$ denotes matrix transpose, $\mathrm{xk}$, and $\mathrm{yk}$ are the Cartesian target coordinates at time index k and $v_{x_{k}}$ and $v_{y_{k}}$,are their respective derivatives (velocities).The state equation for the target motion could be approximated with a linear equation of the form

$$
X_{k+1}=F_{k} x_{k}+G w_{k}
$$

Where $x_{k}$ is the state vector that contains state variables at time $\mathrm{k}$, and $w_{k} \sim N\left(0, Q_{k}\right)$ which is assumed as zero mean white Gaussian noise with covariance $Q_{k}$ (called process noise). 
The state equation for the two dimensional target motion could be approximated with a linear equation of the form

$$
\begin{aligned}
{\left[\begin{array}{l}
x_{k+1} \\
y_{k+11} \\
v_{x_{k+1}} \\
v_{y_{k+1}}
\end{array}\right]=} & {\left[\begin{array}{llll}
1 & 0 & t_{k} & 0 \\
0 & 1 & 0 & t_{k} \\
0 & 0 & 1 & 0 \\
0 & 0 & 0 & 1
\end{array}\right]\left[\begin{array}{l}
x_{k} \\
y_{k} \\
v_{x_{k}} \\
v_{y_{k}}
\end{array}\right] } \\
& +\left[\begin{array}{ll}
t_{k} & 0 \\
0 & t_{k} \\
1 & 0 \\
0 & 1
\end{array}\right]\left[\begin{array}{l}
w_{x} \\
w_{y}
\end{array}\right]
\end{aligned}
$$

Comparing "(2)" and "(3)", then process noise covariance matrix can be written as

$$
Q=E\left[w_{k} w_{k}^{T}\right]=\int_{0}^{T} G \sigma^{2} G^{T} d t
$$

Where $\sigma$ is the standard deviation of the Gaussian random noise. Which can be given as

$$
\left[\begin{array}{cccc}
q_{x} * \frac{t_{k}^{3}}{3} & 0 & q_{x} * \frac{t_{k}^{2}}{2} & 0 \\
0 & q_{y} * \frac{t_{k}^{3}}{3} & 0 & q_{y} * \frac{t_{k}^{2}}{2} \\
q_{x} * \frac{t_{k}^{2}}{2} & 0 & q_{x} * t_{k} & 0 \\
0 & q_{y} * \frac{t_{k}^{2}}{2} & 0 & q_{y} * t_{k}
\end{array}\right]
$$

Where

$$
\begin{aligned}
& \begin{array}{l}
q_{x}=\text { level of power spectral density of X-directional } \\
\text { noise in } \mathrm{m} / \mathrm{sec}^{2}
\end{array} \\
& \qquad \begin{array}{l}
q_{y} \\
=\text { level of power spectral density of Y-directional }
\end{array} \\
& \text { noise } \mathrm{m} / \mathrm{sec}^{2}
\end{aligned}
$$

The measurement model of the system can be written as

$$
z_{k}=h\left(x_{k}, v_{k}\right)
$$

Where ${ }^{z_{k}}$ measurement vector, and $v_{k} \sim\left(0, R_{k}\right)$ which is assumed as zero mean white Gaussian noise with covariance $R_{k}$ (called measurement noise covariance). Both noises are assumed to be uncorrelated.

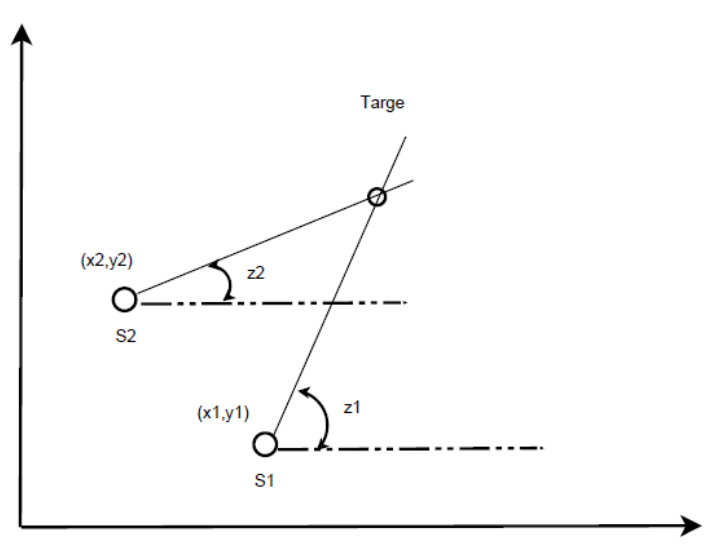

Fig. 2: sensor placement and measurement Scenario

For the above scenario sensors are placed at $\left(s_{x}^{1}, s_{y}^{1}\right),\left(s_{x}^{2}, s_{y}^{2}\right)$ and measurement equation can be written as

$$
\begin{aligned}
{\left[\begin{array}{l}
\theta_{1} \\
\theta_{2}
\end{array}\right]=} & {\left[\begin{array}{l}
\tan ^{-1}\left(y_{k}-s_{y}^{1}\right) /\left(x_{k}-s_{x}^{1}\right) \\
\tan ^{-1}\left(y_{k}-s_{y}^{2}\right) /\left(x_{k}-s_{x}^{2}\right)
\end{array}\right] } \\
& +\left[\begin{array}{c}
s_{d} * \text { randn } \\
s_{d} * \text { randn }
\end{array}\right]
\end{aligned}
$$

Where $\mathrm{Sd}$ is the standard deviation of the measurement noise.

Measurement noise covariance can be written as

$$
R_{k}=E\left(v_{k} v_{k}^{T}\right)
$$

This can be written as

$$
R_{k}=\operatorname{dig}(s d, s d)
$$

The accuracy of the estimation depends on the priori measurement noise covariance matrix $R_{k}$ and process noise covariance matrix $Q$ which interns depends upon these spectral densities $\mathrm{q}_{\mathrm{x}}$ and $\mathrm{q}_{\mathrm{y}}$. Selecting optimum parameters of these values gives optimum performance of the filter.

Trial and error approach to obtain these the above said three tuning parameters is tedious process and doesn't guarantee the accuracy of estimation in Mean Square Error (MSE) sense. Choosing optimum Parameters of noise covariance matrices, "i.e." is tuning the filter is a challenging task for Kalman filter designer. 
In this paper another approach of tuning the Unscented Kalman Filter based on the swarm intelligence and hybrid approach is proposed.

\section{Interactive Multiplemodels}

The target is maneuvering and switching among different models like constant velocity (CV), constant acceleration (CA) or constant turn (CT), Interactive Multiple Models (IMM) are employed. The main idea of the IMM algorith $m$ is to weigh the estimates fro $m$ the filters matched to the different modes. Different modes have different state space models. The weights are based on the time variant mode probabilities that imply how close the estimate from each filter is to the corresponding model [13].

Interactive Multiple Model-Extended Kalman Filter (IMM-EKF) and Unscented Kalman Filter (IMM-UKF) have been developed to acknowledge the insensitivity of Kalman Filter towards target maneuvering. The IMM-EKF and IMM-UKF both use several target motion models. The choice of the target motion model is one of the issues in designing target tracking system. The performance of the EKF and UKF degrades rapidly if the target is executing manuever. To solve this problem, IMM-EKF and IMM-UKF uses several possible models for the target motion (Like Constant velocity, Constant acceleration etc) and a probabilistic switching between these models.

\section{A. Structure of the IMM Algorithm}

In each time step, the IMM algorithm performs four steps to yield the overall state estimates:

1. Interacting or mixing of the estimates: from the estimate, $\hat{x}_{i}(k-1 \mid k-1)$ and mode probability, $\mu_{i}(k-1)$ of each filter in the previous step, obtain the mixing estimate, $\hat{x}_{i}^{o}(k-1 \mid k-1)$ and covariance, $P_{i}^{o}(k-1 \mid k-1)$ under the assumption that a particular mode is in effect at the present time. The mixing estimate is used as an initial state in current step.

2. Model-conditional filtering: using the Kalman filter, update state and covariance, $\hat{x}_{i}(k \mid k), P_{i}(k \mid k)$.

3. Mode probability update: using the likelihood function, update the mode probability, $\mu_{i}(k)$.

4. Combination of estimates: based on the mode probability, weigh the estimate from each filter and combine them, $\hat{x}(k \mid k), P(k \mid k)$.

EKF or UKF filters can be used in place of filters and run parallel as shown in figure 3. Then it is called IMMEKF or IMM-UKF filters.

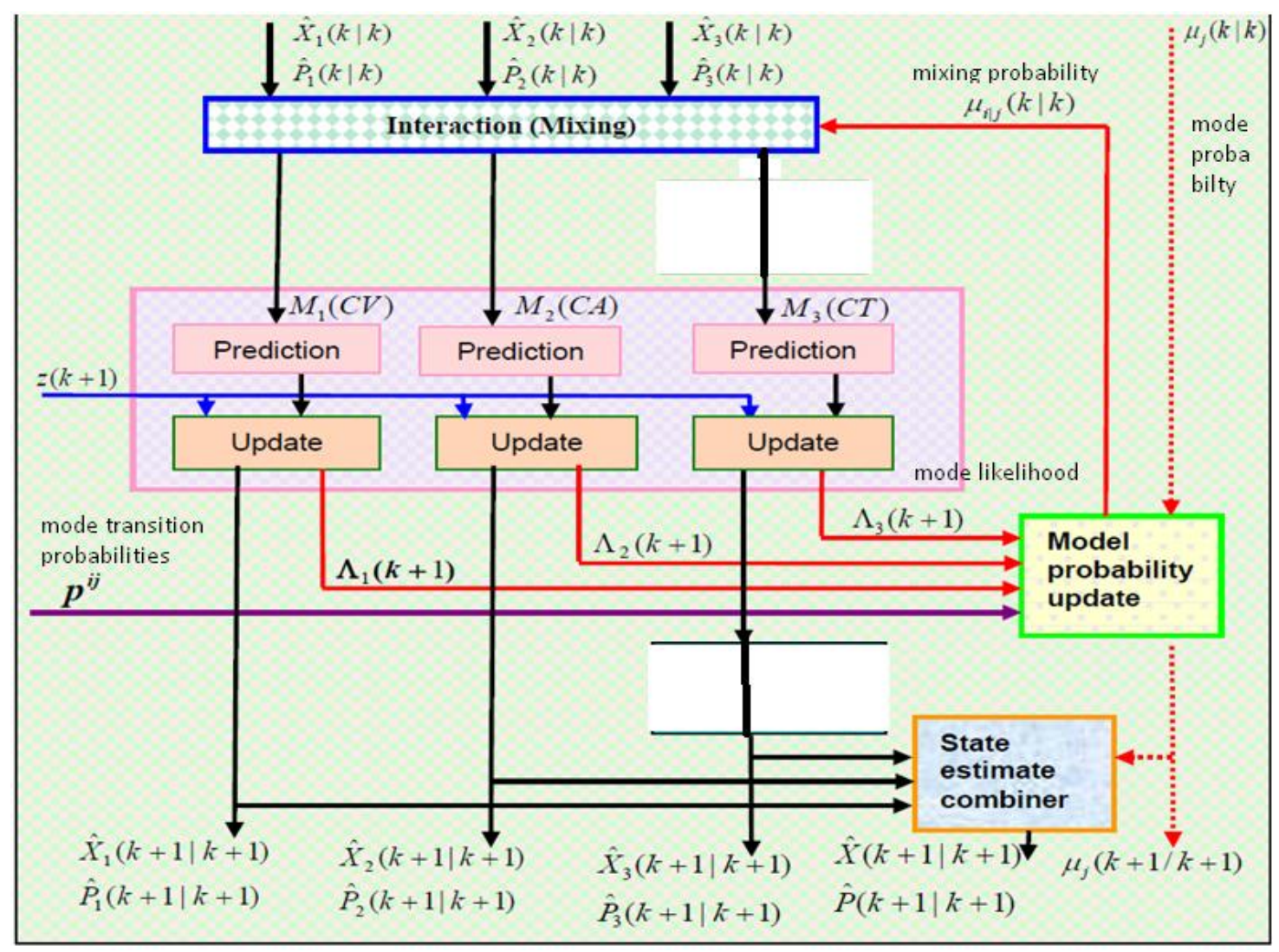

Fig. 3: IMM- Filter Structure 


\section{Tuning of IMM-EKF, IMM-UKF}

Tuning of the filter is referred as estimation of the noise covariance matrices. It has been shown previously that the performance of an IMM-EKF and IMM-UKF filter depends largely on the accuracy of the knowledge of process covariance matrix and measurement noise covariance matrix. Incorrect apriori knowledge of noise covariance may lead to performance degradation and it can even lead to practical divergence. Hence, intelligent method of estimation of these matrices becomes very important. Measurements can be performed before the operation of the filter under various noise conditions and measurement noise covariance can be obtained off line [15]. For tuning of Kalman filters least square method is generally used [16-17].

\section{A. Genetic Algorithm}

Genetic algorithm is a powerful evolutionary computing tool developed by Goldberg. Its main principal is "Select the Best, Discard the Rest" as adopted by naturally in the environment. Two important elements required for any problem before a genetic algorithm can be used for a solution are:

1) Method for representing a solution (encoding)

Ex: string of bits, numbers, and character

2) Method for measuring the quality of any

Proposed solution, using fitness function

Ex: Determining RMSE

The space of all feasible solutions (it means objects among those the desired solution is) is called search space (also state space). Each point in the search space represents one feasible solution. Each feasible solution can be "marked" by its value or fitness for the problem. The whole process can be categorized into following sub processes [18-19].

\section{1) Initialization}

Initially many individual solutions are randomly generated to form an initial population, covering the entire range of possible solutions (the search space).Each point in the search space represents one possible solution marked by its value (fitness)

\section{2) Selection}

A proportion of the existing population is selected to bread a new breed of generation.

3) Reproduction Generate a second generation population of solutions from those selected through genetic operators: crossover and mutation.

4) Termination- A solution is found that satisfies prespecified criteria
5) Fixed number of generations.-The highest ranking solution fs fitness has reached. The following flow chart pictorially represents Genetic algorithm in a brief and efficient manner.

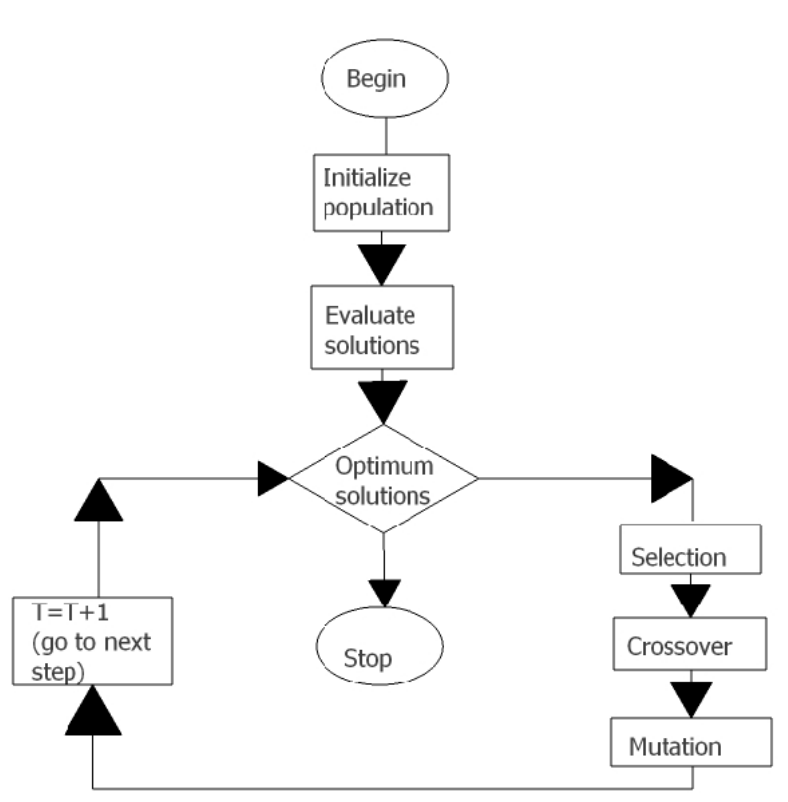

Fig. 4: Flow Chart of Genetic algorithm

Disadvantages of genetic algorithm

* Computation complexity is high

* More training time is required

* Conversion from binary to decimal

* Chances of falling to local minima

\section{B. Particle swarm optimization}

PSO is population based stochastic optimization technique inspired by social behavior of bird flocking or fish schooling etc [19-20]. The swarm of particles indicates estimation of multiple parameters involved in the problem. We can begin with initializing a random swarm of particles. During each iteration fitness of the particle is evaluated with the help of fitness function.

The trajectory of the particle is dependent on three factors: its previous position, pbest and gbest. Greater the strain of particle in searching food, smaller is the acceleration coefficients. The inertial weight factor $\mathrm{w}$ signifies the importance of the particle's previous position in further search.

Velocity updation

$$
\begin{aligned}
v_{i}(t+1)= & w \cdot v_{i}(t) \\
& +c_{1} \operatorname{rand}(\operatorname{pbest}(t)-x(t)) \\
& +c_{2} \cdot \operatorname{rand} \cdot\left(\operatorname{gbest}(t)-x_{i}(t)\right)
\end{aligned}
$$


Position updation

$$
\mathrm{P}=\mathrm{P}+\mathrm{V}
$$

Where

$\mathrm{P}$ - Instantaneous position of the particle

V - Instantaneous velocity of the particle

Pbest - positional best of the particle

gbest-global best position of the swarm of the particles

$$
\begin{aligned}
& \mathrm{W}-\text { Inertial weight factor } \\
& \mathrm{C} 1, \mathrm{C} 2 \text { - acceleration coefficients }
\end{aligned}
$$

Thus each particle tends to move towards gbest to reach food early. If gbest has less number of values then the particles will reach food early. The algorith m comes to an end when all the particles converge at the gbest i.e. food position [8]. In our problem i.e. attaining minimum possible value for steady state error signal is considered as global optimum.

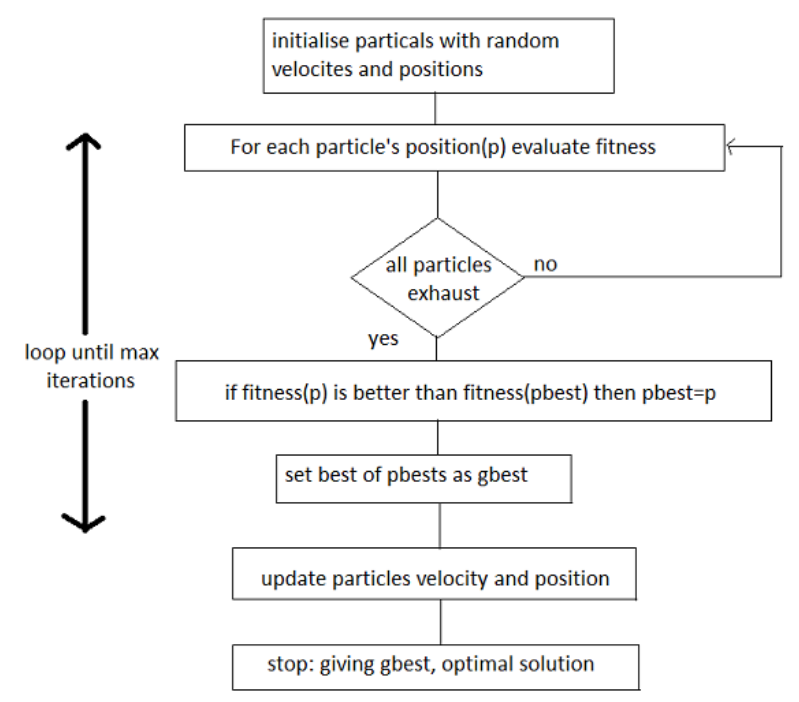

Fig. 5: Flow chart for PSO Algorithm

\section{Hybrid GA-PSO Algorithm}

One advantage of PSO over GA is its zlgorithmic simplicity. Another clear difference between PSO and GA is the ability to control convergence in PSO. The main problem with PSO is that it prematurely converges (Van den Bergh and Engelbrecht 2004) to stable point, which is not necessarily global extreme To overcome the limitations of PSO and GA, hybrid algorithm is proposed. Such approach is expected to combine merits of PSO and GA in tuning gain para meters. The merit of GA lies in its genetic operator, crossover and mutation. By applying crossover operation, information can be swapped between two partic les to have the ability to fly to the new search area and mutation operator increases the population .The total numbers of iterations are equally shared by GA and PSO. First half of the iterations are run by GA and the solutions are given as initial population of PSO. Remaining iterations are run by PSO [11, 21].

\section{Applying GA-PSO in Filter Tuning}

We refer to filter tuning as a process of obtaining parameters of a filter such as values of matrices $Q$ and $\mathrm{R}$ for UKF that give the best filter performance in Mean Square Error (MSE) sense. Typically this kind of problems of designing a filter with optimal tuning parameters was left up to engineering intuition, and trial and error method that do not guarantee best filter performance due to large number of parameters to be tuned. A straightforward way of tackling this problem is to employ global optimization method that minimizes function of MSE position error with respect to filter parameters. There are several issues associated with such an approach. First, each time we need a value of MSE during global optimization procedure we have to run IMM_EKF and IMM-UKF on all available data. This requires a significant computational time since for example in order to find a global minimu m of a smooth function of 3 parameters; we need to compute the function value many times [22-24].

Here in this problem we are tracking with constant velocity and with small manoeuvre such as to relate practical problem. Therefore we have two power spectral densities of the corresponding continuous process noise, one parameter of measurement noise standard deviations (bearing). So a total of three parameters have to be optimized. Taking the extreme worst cases of these three parameters, we precede according to the above mentioned optimisation algorithms.

\section{Simulations and Results}

Here we consider a target scenario in which a moving target in the scene and two angular sensors for tracking it. The sensors are placed at $\left(s_{x}^{1}, s_{y}^{1},\right)=(-1 m,-2 m)$ and $\left(s_{x}^{2}, s_{y}^{2},\right)=(-1 m,-2 m)$. The measurement noise standard deviation is taken as $\mathrm{Sd}=0.5$ radians and spectral densities of the process noise is consider as $\mathrm{q}$ $\mathrm{x}=0.1$ and $\mathrm{qy}=0.1$ to generate data as show "Fig.4" below. The simulations are performed using industry standard MATLAB and EKF/UKF Toolbox. The following figure gives Radar sensor measurements 


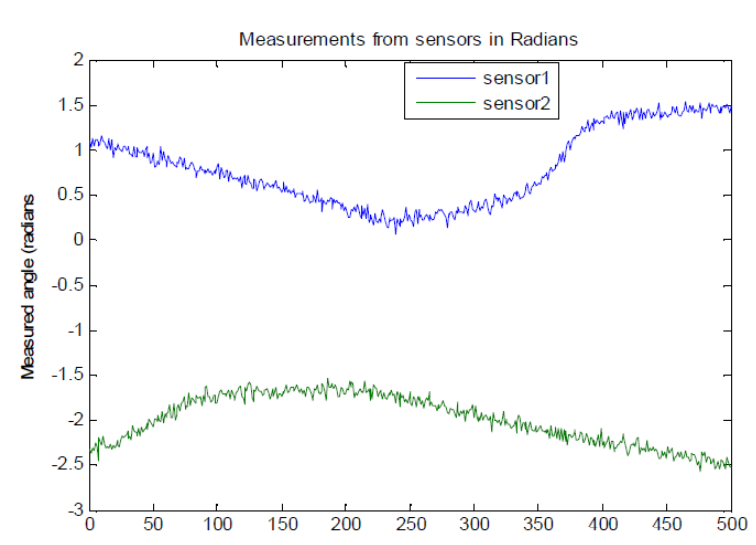

Fig. 6: Radar observations

Figure6: IMM-EKF filtering results for path1

\section{GA-Parameters}

GA type used=Standard Genetic Algorithm

Number of Variables $=3$
Initial Population size $=25$

Length of Chromosome $=20$

Probability of Crossover $=0.8$

Probability of Mutation $=0.2$

\section{Initialization of PSO}

Size of the $\mathrm{s}$ warm " no of birds $=30$;

Maximum number of "birds steps $=30$;

Dimension of the problem $=3$;

PSO parameter $\mathrm{C} 1=2.05$

PSO parameter $\mathrm{C} 2=2.05$

Pso momentum or inertia $\mathrm{w}=0.45$

Search space for $\mathrm{Sd}=0$ t0 0.1

Search space for $\mathrm{qx}=0 \mathrm{t} 00.01$

Search space for $\mathrm{qy}=0 \mathrm{t} 00.05$

\section{Filtering result with IMM-EKF}

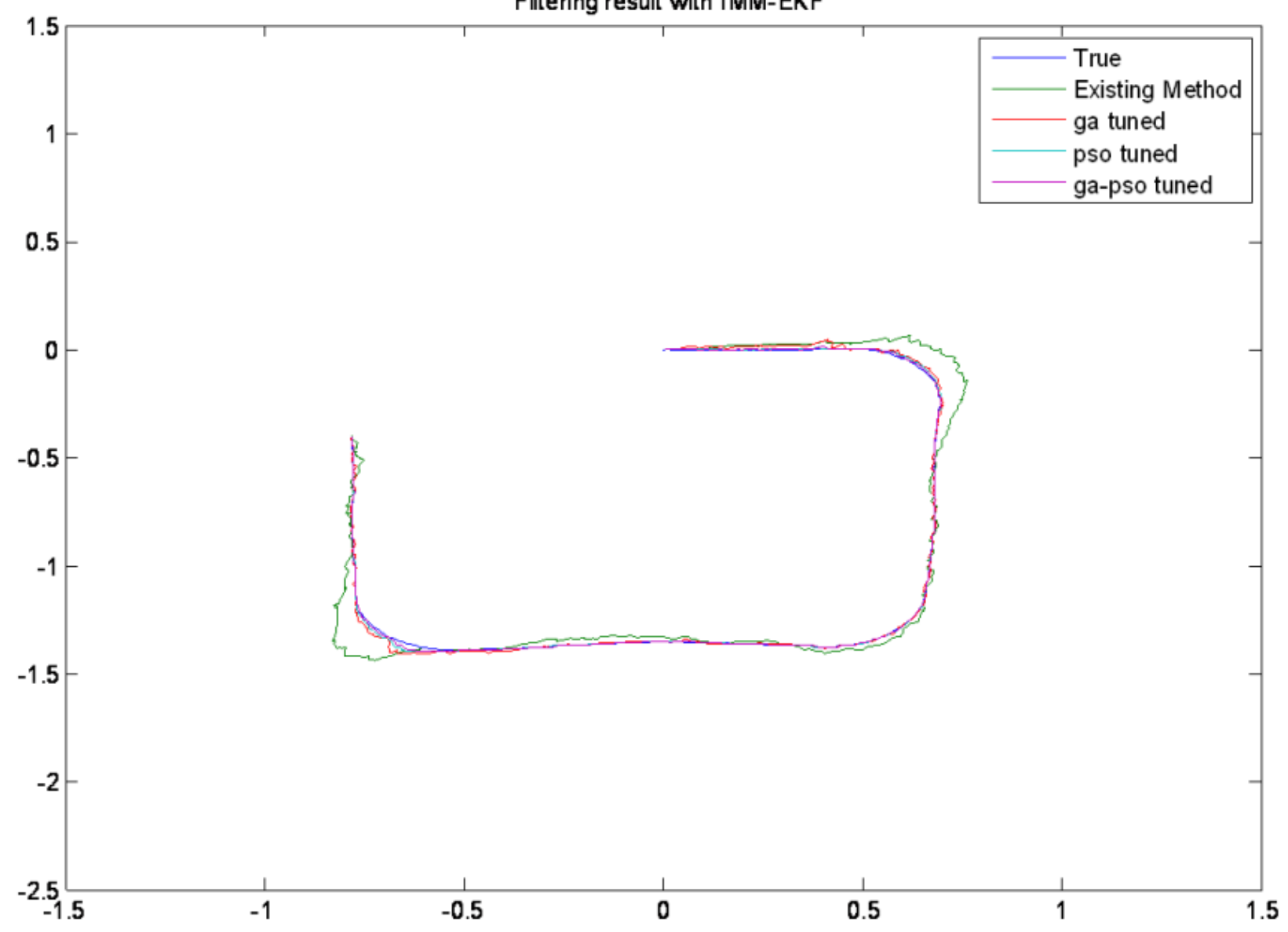

Fig. 7: IMM-EKF Filtering results for trajectory 1 


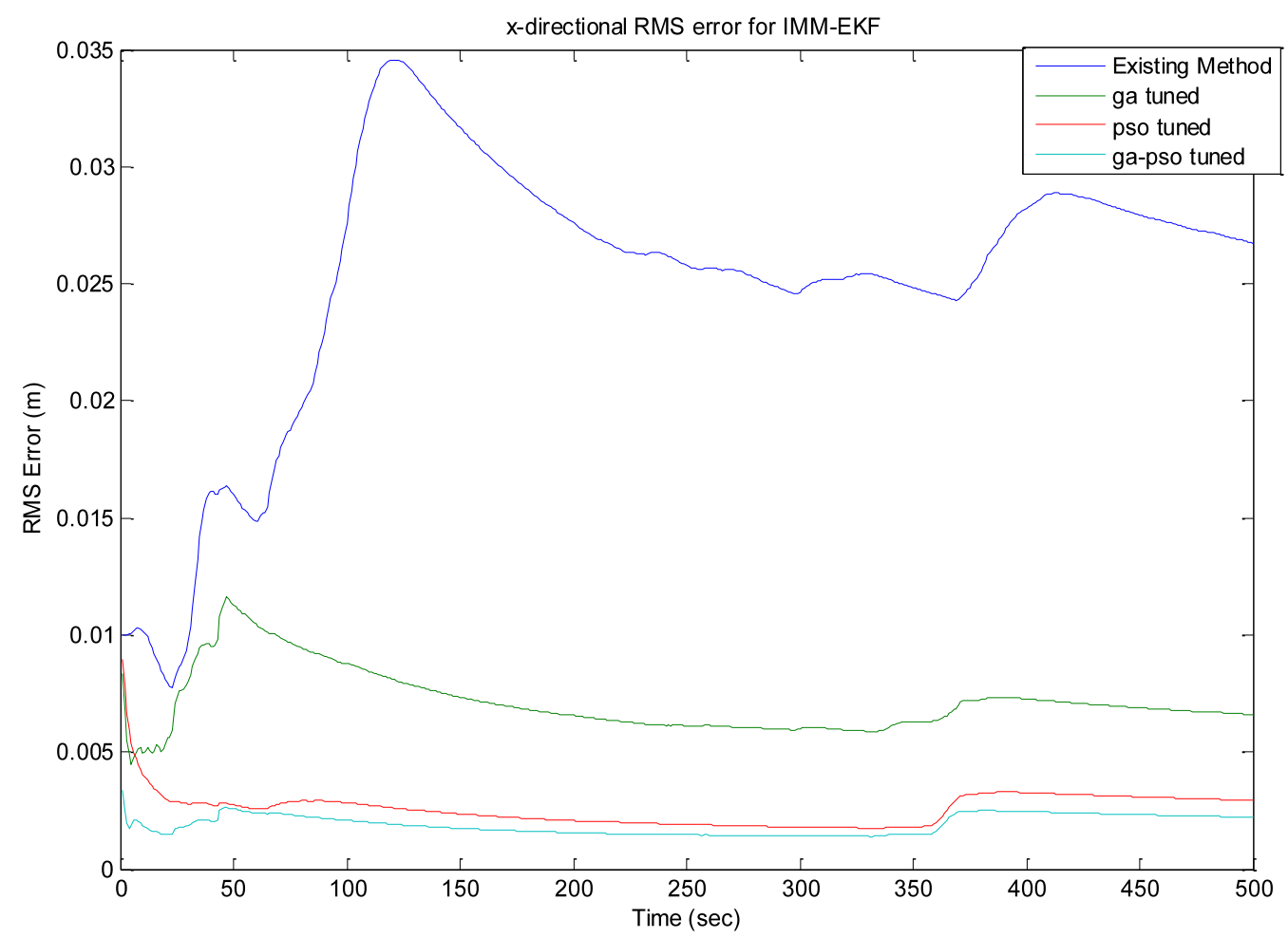

Fig. 8: X-directional RMS Error IMM-EKF trajectory 1

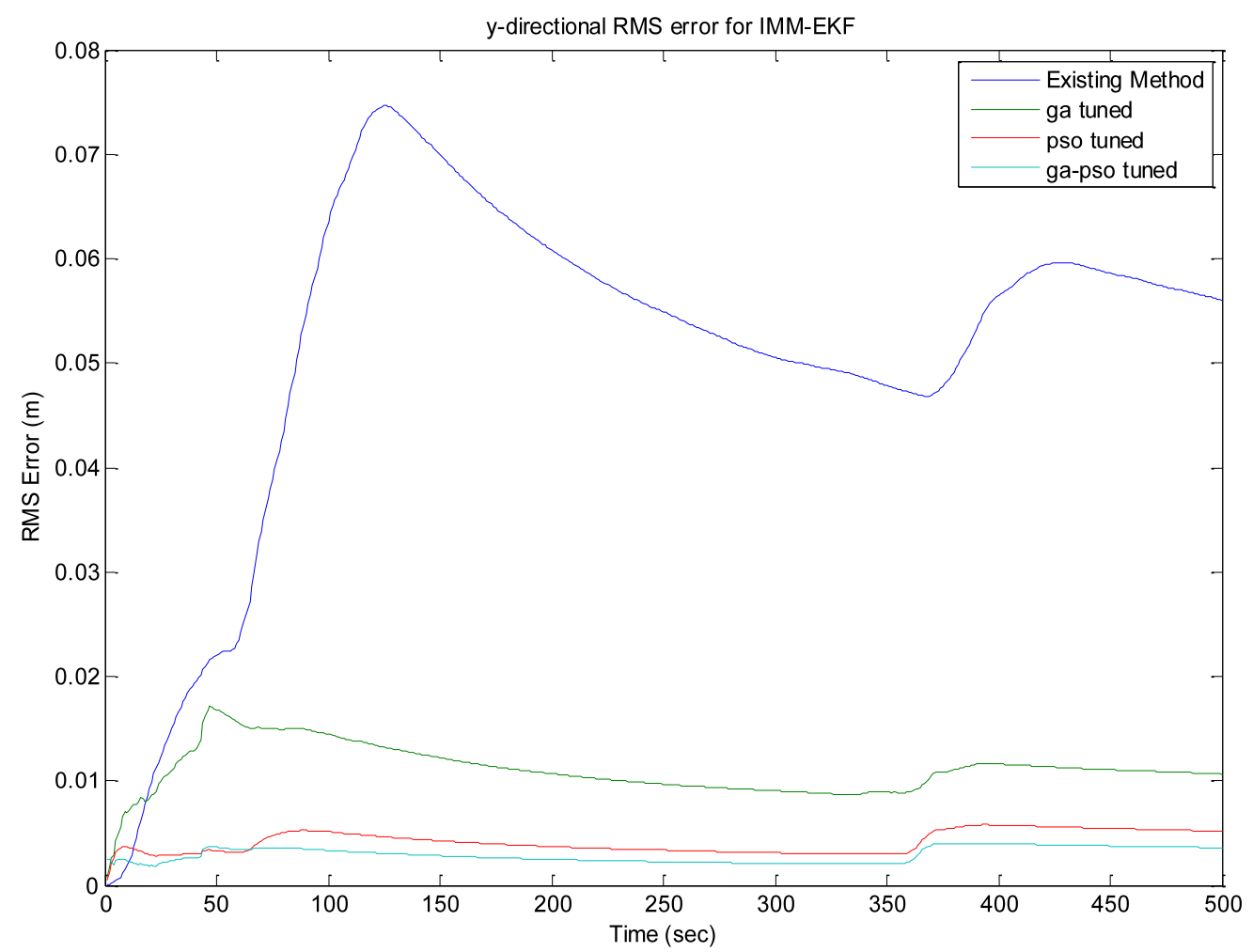

Fig. 8: Y-directional RMS Error IMM-EKF trajectory 1

"Fig 7 and 8" shows RMSE for IMM-EK filter. From this we can say that GA-PSO tuned IMM-EKF is performing better and errors are listed in Table 1 in meters with different tuning methods. 
Table 1: Filtering results of IMM-EKF for trajectory 1

\begin{tabular}{|l|c|c|}
\hline \multicolumn{1}{|c|}{ TUNING METHODS } & X-directional Error $(\mathbf{m})$ & Y-directional Error $(\mathbf{m})$ \\
\hline Existing method & 0.0267 & 0.0560 \\
\hline Genetic algorithm tuning & 0.0066 & 0.0106 \\
\hline Particle swarm optimization tuning & 0.0029 & 0.0052 \\
\hline Genetic algorithm and particle swarm optimization tuning & 0.0022 & 0.0036 \\
\hline
\end{tabular}

The following "Fig.9" shows Filtering results with IMM-UKF filter.

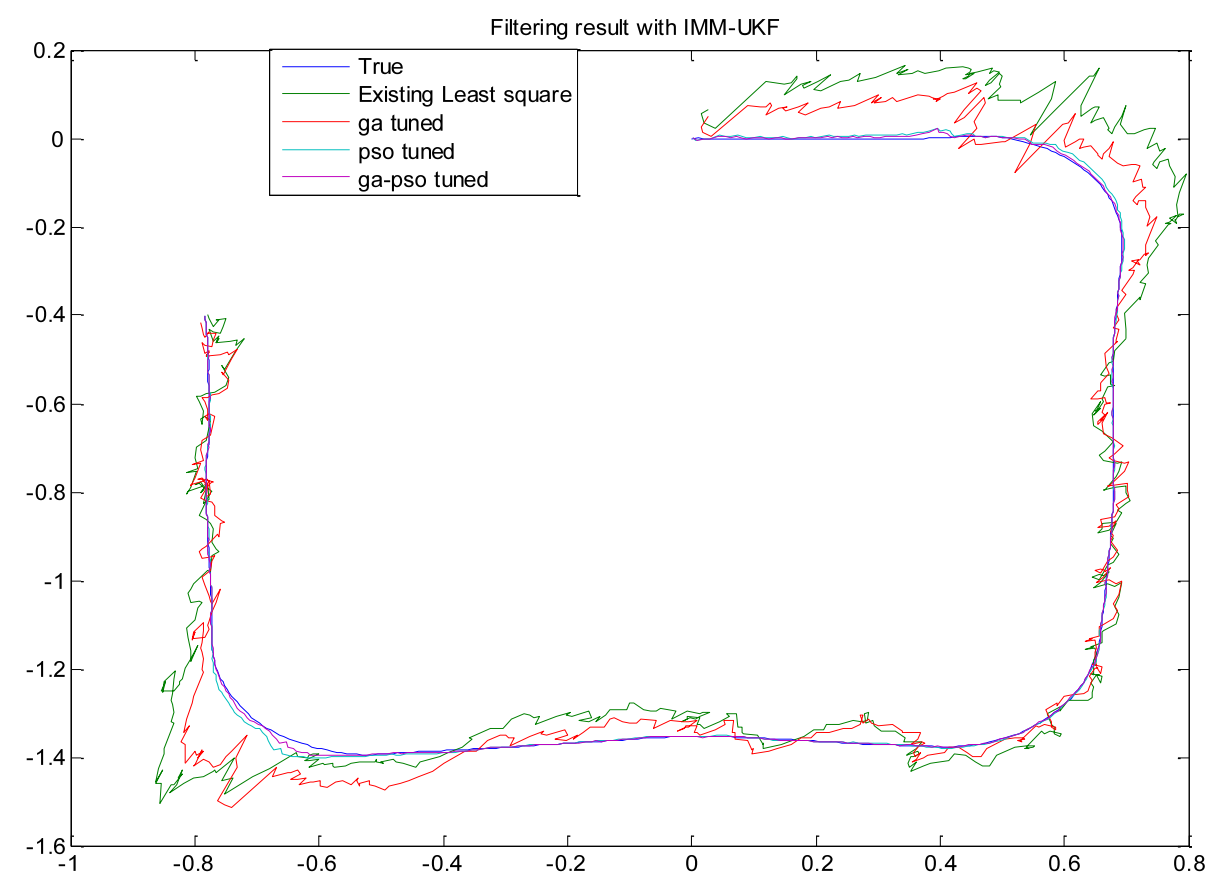

Fig. 9: IMM-UKF filtering results for path1

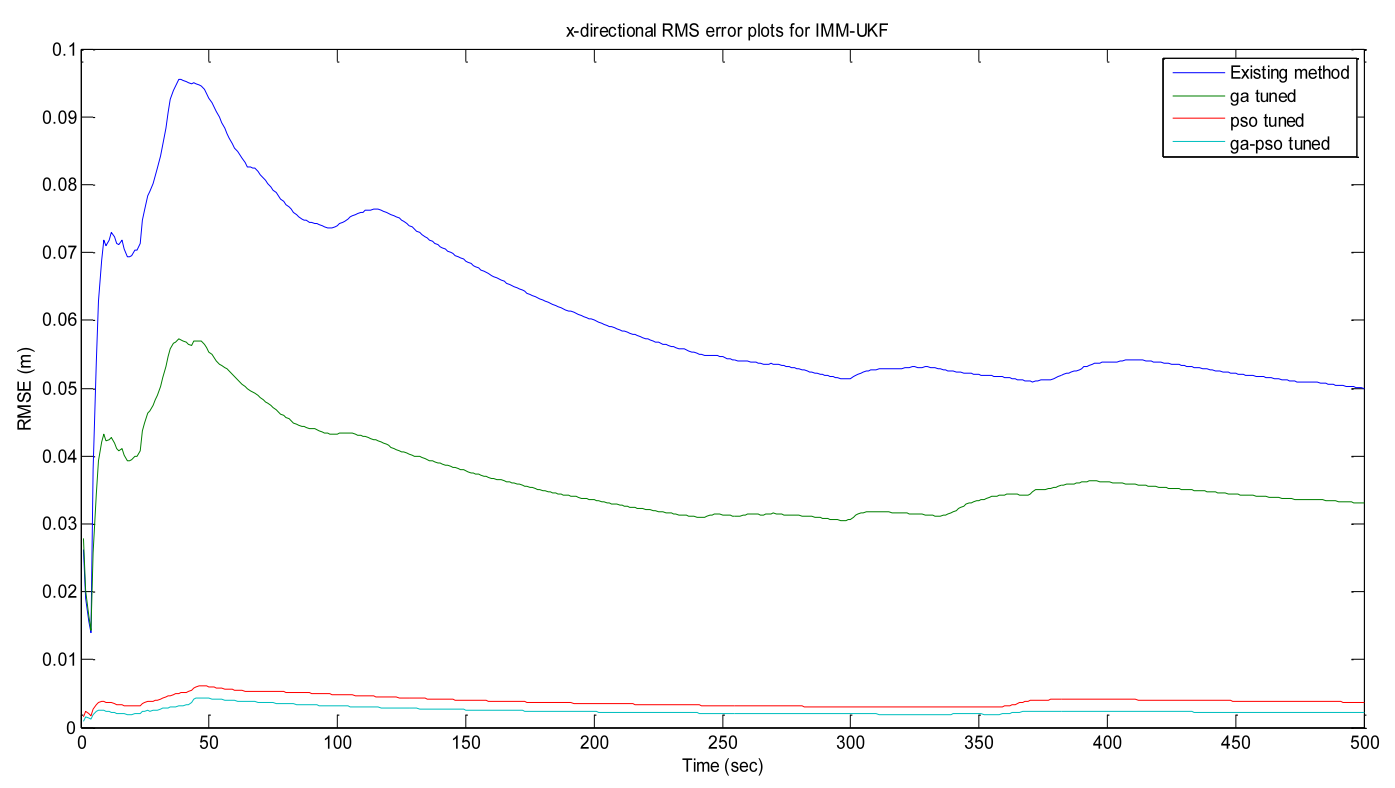

Fig. 10: X-directional RMS Error 


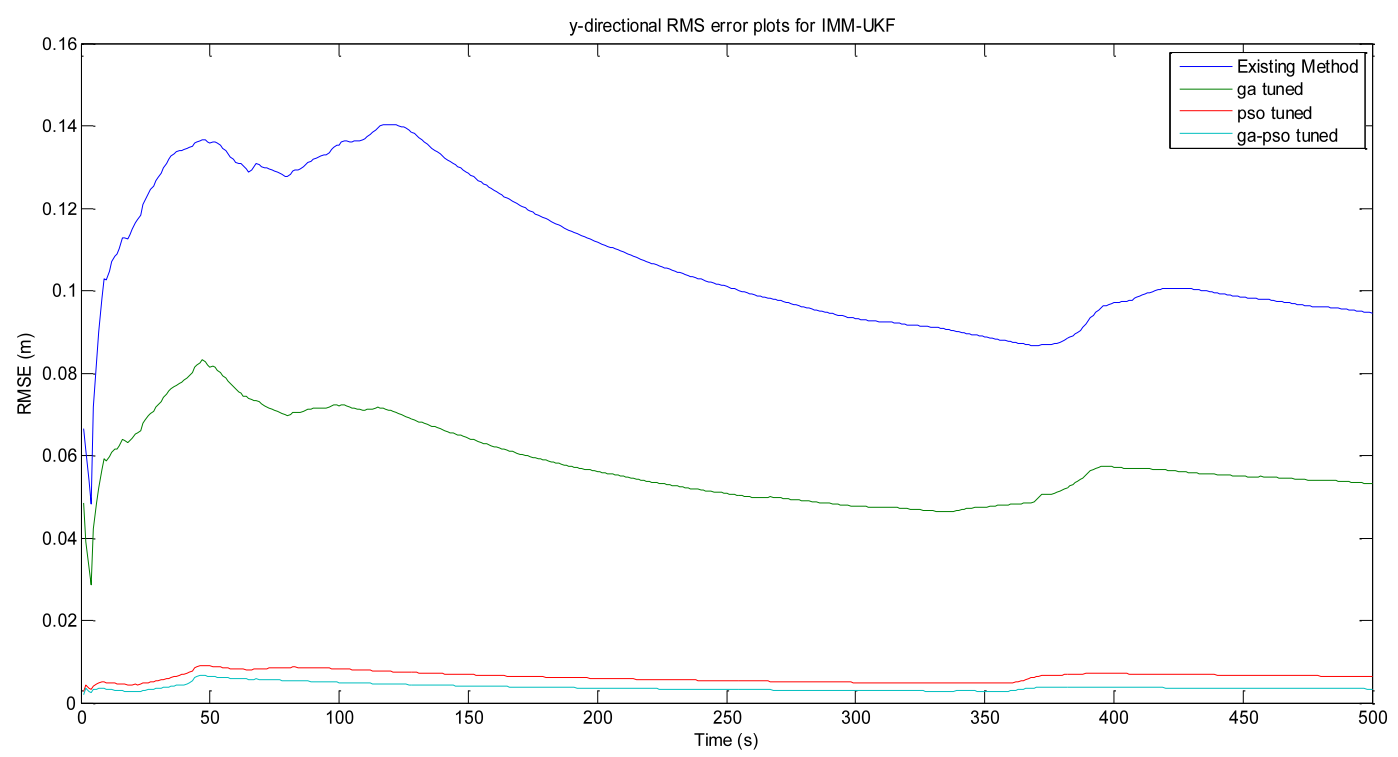

Fig. 11: X-directional RMS Error

"Fig.10 and 11" shows RMSE for IMM-UK filter. From this we can say that GA-PSO tuned IMM-UKF is performing better and errors are listed in Table 2 in meters with different tuning methods

Table 2: Filtering results of IMM-UKF for trajectory 1

\begin{tabular}{|l|c|c|}
\hline \multicolumn{1}{|c|}{ Tuning Methods } & X-Directional RMS Error (M) & Y-Directional RMS Error (M) \\
\hline Trial \& Error & 0.0500 & 0.0945 \\
\hline Genetic Algorithm Tuning & 0.0330 & 0.0532 \\
\hline Particle Swarm Optimization Tuning & 0.0037 & 0.0064 \\
\hline Genetic Algorithm And Particle Swarm Optimization Tuning & 0.0021 & 0.0034 \\
\hline
\end{tabular}

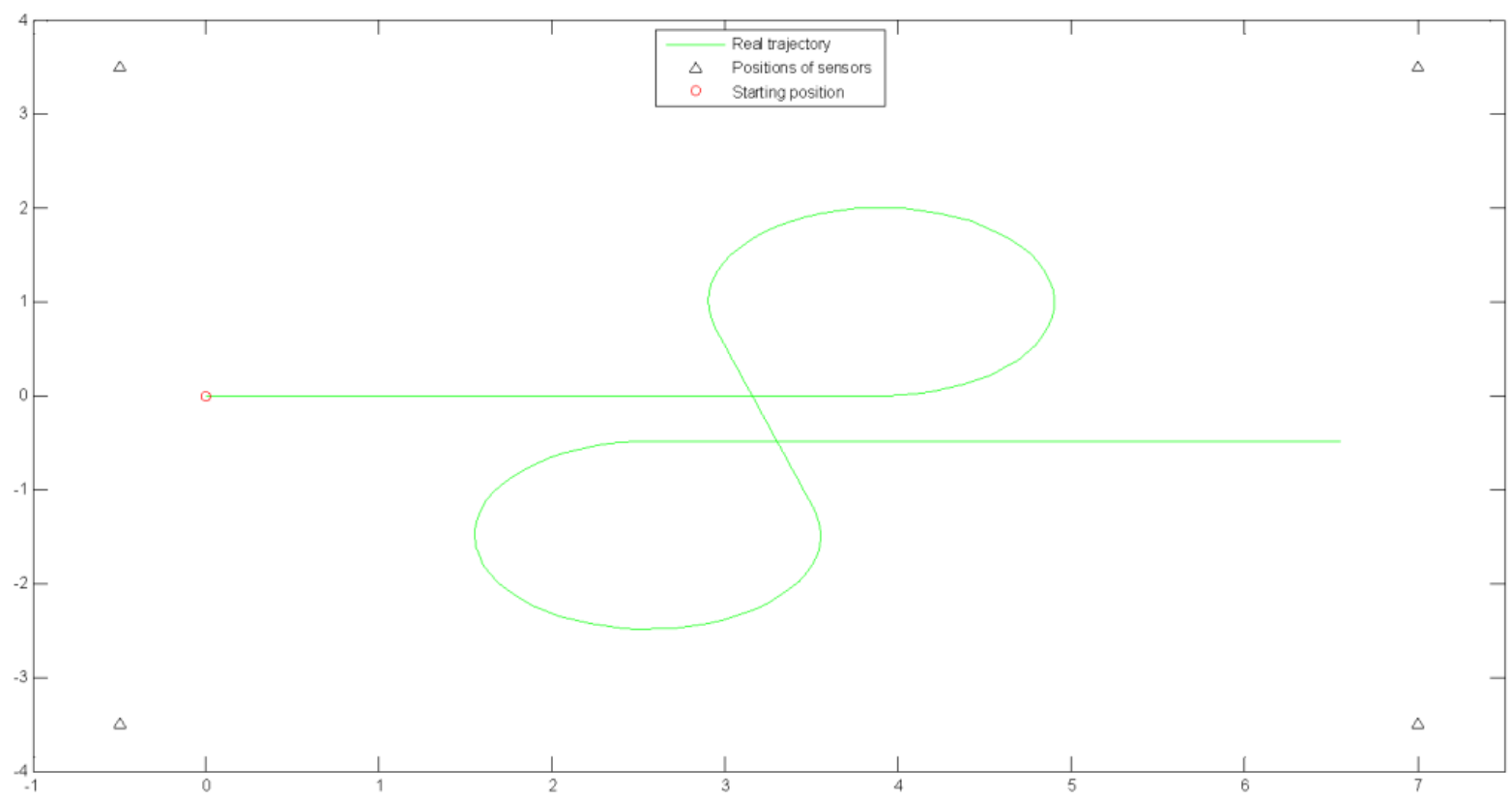

Fig. 12: true trajectory for path 2

From the above table II we can say that the Hybrid GA-PSO tuned Kalman filter performing better compare red to conventional tuning. The trajectory 2 is a target flying in the $x-y$ plane starting with an initial 
position and with an initial velocity $[1 \mathrm{~m} / \mathrm{s}$ 0] executes a 5- motion sequence (CV-CT-CV-CT-CV).

1. CV for the first $4 \mathrm{sec}($ time intervals $1-40)$

2. CT for the next $5 \mathrm{sec}($ time intervals 41-90)

3. CV for the next 2 sec(time intervals 91-110)

4. CT for the next $5 \mathrm{sec}($ time intervals 111-160)

5. CV for the next $4 \mathrm{sec}($ time intervals 161-200)

\section{Parameters:}

6. No of models: CV model, CT model

7. Process noise covariance: $(0.1,0.5)$

8. Standard deviation of measurement noise: 0.01

9. No of data points $=200$

10. Sampling time $=0.1 \mathrm{sec}$

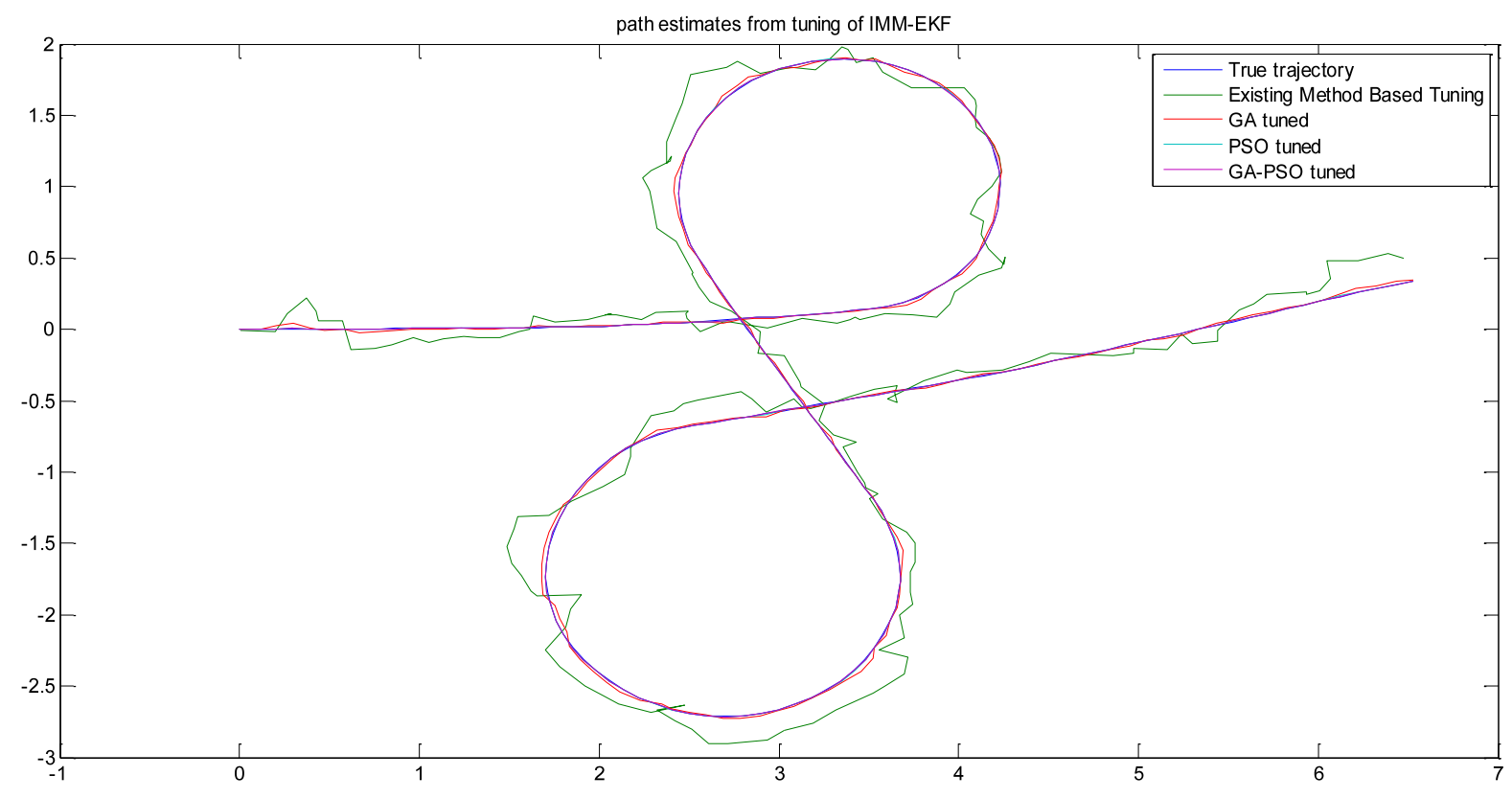

Fig. 13: Filter estimations with conventional and Hybrid Tuning

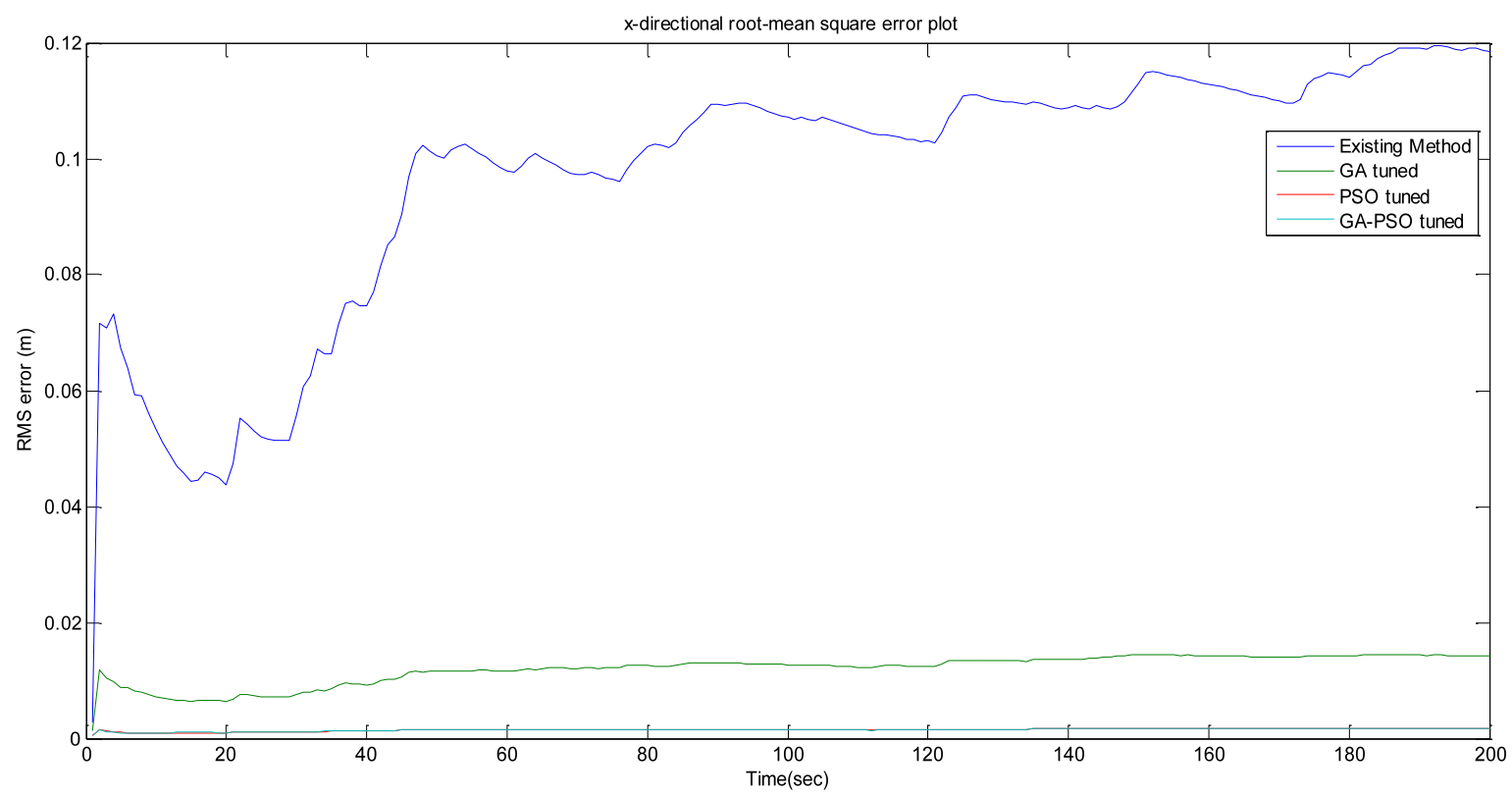

Fig. 14: X-directional RMS Error IMM-EKF for trajectory 2 


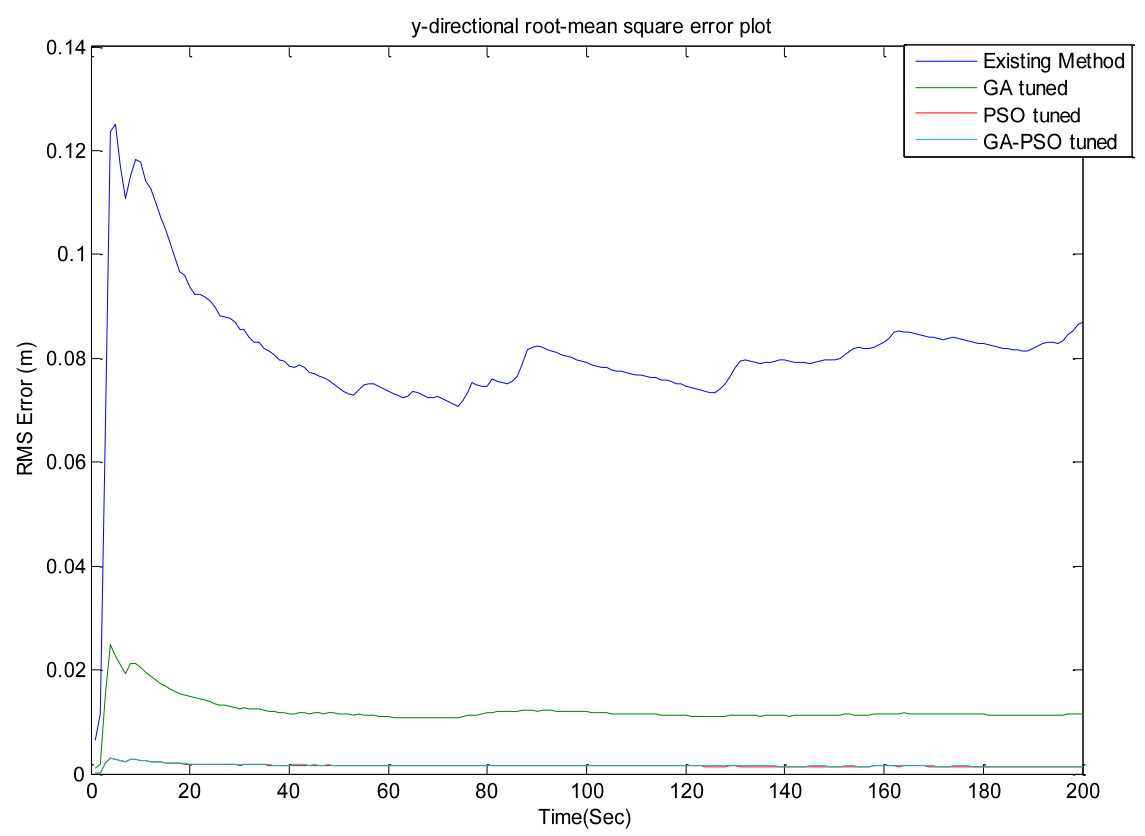

Fig. 15: Y-directional RMS Error IMM-EKF for trajectory 2

"Fig.14 and 15" shows RMSE for IMM-EK filter. From this we can say that GA-PSO tuned IMM-EKF is performing better and errors are listed in Table 3 in meters with different tuning methods

Table 3: Filtering results of IMM-EKF for trajectory2

\begin{tabular}{|l|c|c|}
\hline \multicolumn{1}{|c|}{ Tuning Methods } & X-Directional RMS Error (M) & Y-Directional RMS Error (M) \\
\hline Existing method & 0.1186 & 0.0871 \\
\hline Genetic algorithm tuning & 0.0142 & 0.0116 \\
\hline Particle swarm optimization tuning & 0.0017 & 0.0014 \\
\hline Genetic algorithm and particle swarm optimization tuning & 0.0007 & 0.0007 \\
\hline
\end{tabular}

The "Fig.16" shows the IMM-UKF filtering results for trajectory 2

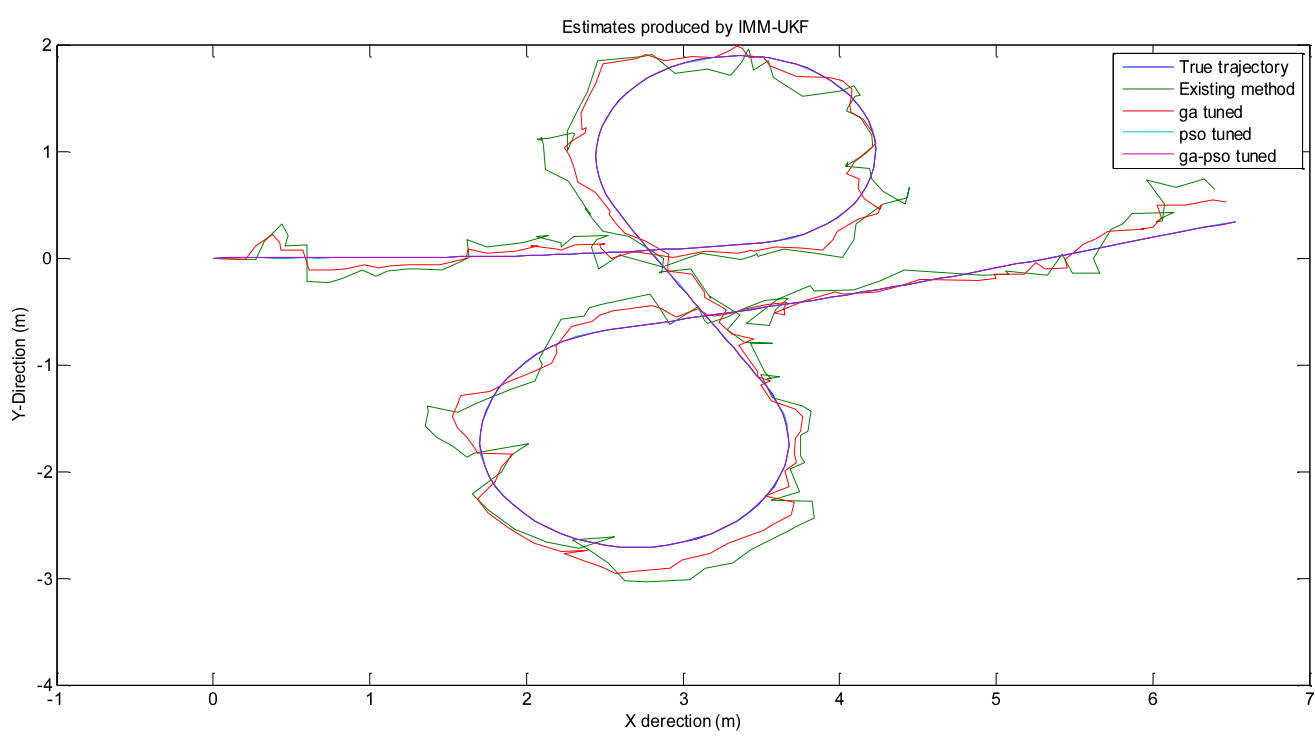

Fig. 16: IMM-UKF filtering results for path2 


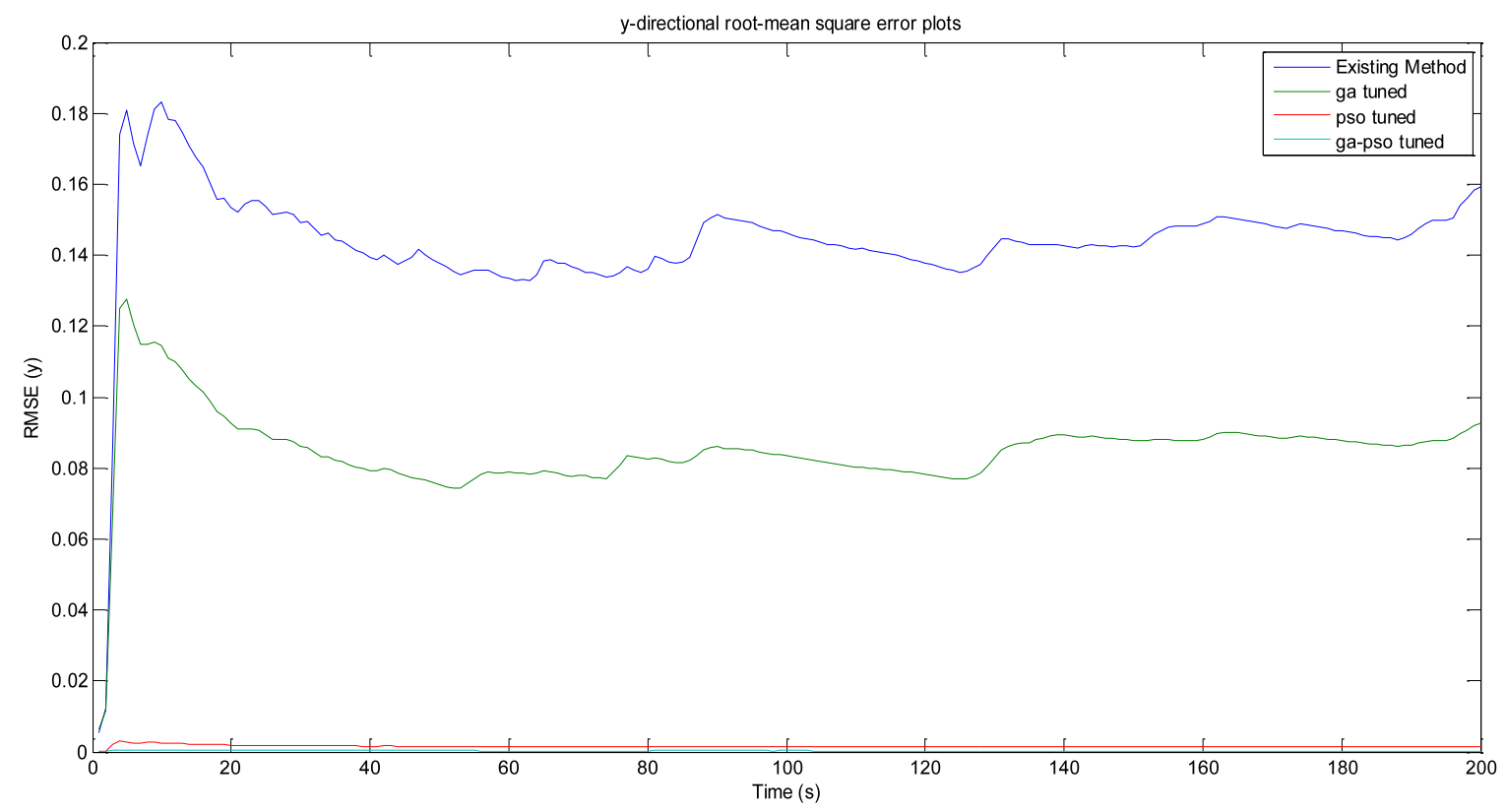

Fig. 17: X-directional RMS Error IMM-UKF for trajectory 2

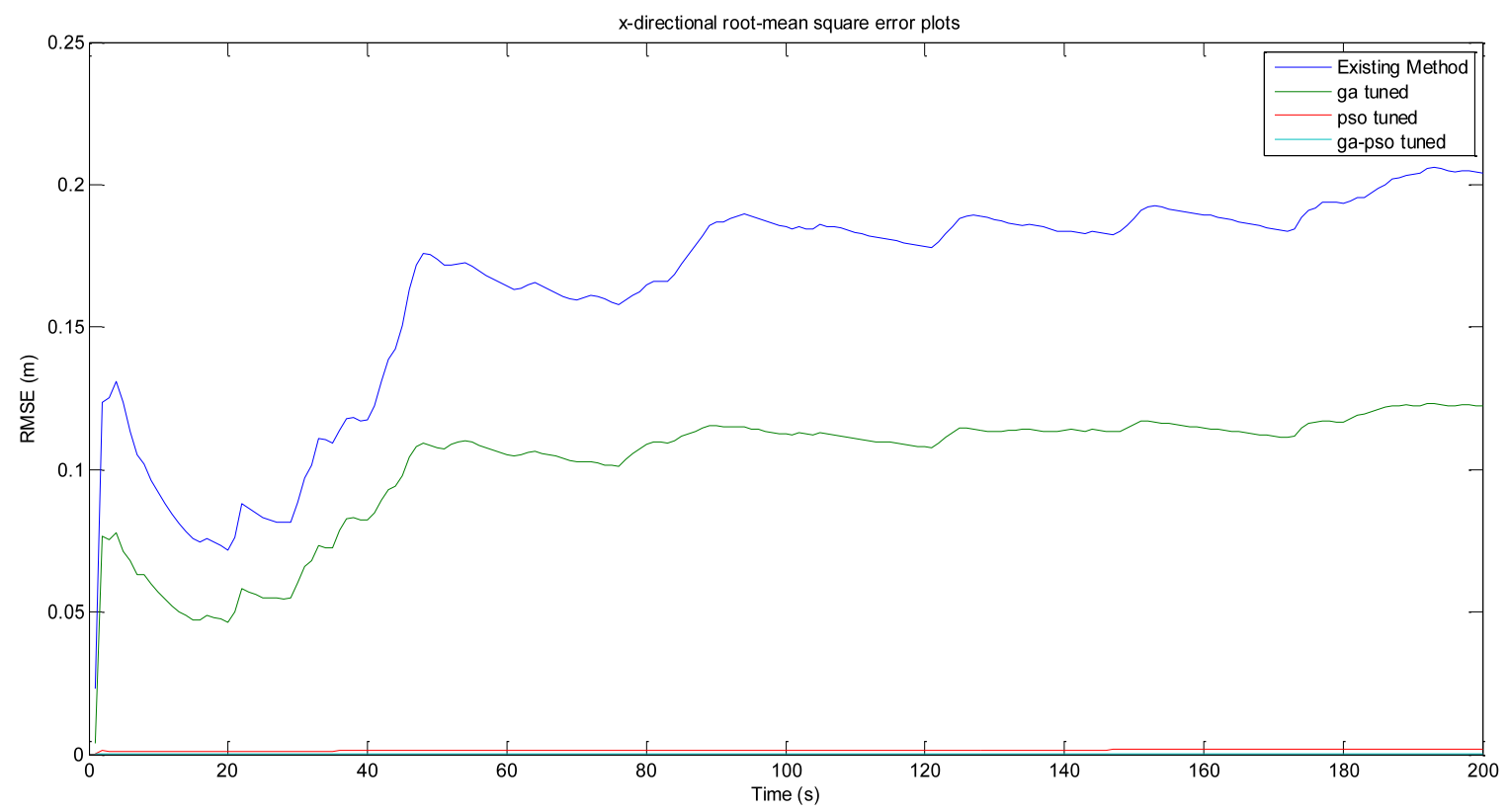

Fig. 18: Y-directional RMS Error using IMM-UKF for trajectory 2

"Fig. 18 and 19" shows RMSE for IMM-UKF. From this we can say that GA-PSO tuned IMM-UKF is performing better and errors are listed in Table 4 in meters with different tuning methods

Table 4: Filtering results of IMM-UKF for trajectory2

\begin{tabular}{|l|c|c|}
\hline \multicolumn{1}{|c|}{ Tuning Methods } & X-Directional RMS Frror $(\mathbf{M})$ & Y-Directional RMS Error(M) \\
\hline Existing method & 0.1152 & 0.0868 \\
\hline Genetic algorithm tuning & 0.1222 & 0.0926 \\
\hline Particle swarm optimization tuning & 0.0017 & 0.0015 \\
\hline Genetic algorithm and particle swarm optimization tuning & 0.0004 & 0.0003 \\
\hline
\end{tabular}


From the above error analysis we can say that Tuned filter in which the covariance matrices are estimated using Nature Inspired Algorithms are giving minimal error.

From the above Table IV shows the RMSE of conventional tuned UKF and Nature inspired Algorithms based tuned UKF. We can see that Hybrid GA-PSO based tuned UKF is giving Minimum RMS error. In case of Computational complexity it is always trade of between computations and RMS error. This can be overcome by latest High speed Digital Signal Processors.

\section{Conclusion}

The paper presents tuning Procedure for IMM-EKF and IMM-UKF. A comparison was made between two non linear filtering algorith $\mathrm{m}$ and tuning of these based on nature inspired evolutionary algorithm for maneuvering target tracking. Since the measurement covariance can be determined in diffe rent environ ments, like off-line, we can get standard deviation for different conditions. Then, Hybrid GA-PSO Tuned IMM-UKF can be applied for fine tuning of noise covariance matrices. The results are shown for conventional tuned and Hybrid GA-PSO-tuned IMM-UKF and we can say Tuned filter gives better performance. Computation complexity can be overcome by $\mathrm{Hi}$ speed DSP processors available.

\section{References}

[1] S.Sadhu,M.Srinivasan and T.Ghosal, "Robustness and Tuning of Bearing only tracking" pp 4249,IE(I) Signal processing ,Journal,vol 87, Dec2006.

[2] L Song and J L Speyer "A Stochastic Analysis of Modified Gain Extended kalman filter With Applications to Estimation with Bearing s Only Measurements, IEEE Transactions on Automatic Control, , pp 940-949 vol 30,no 10,1985 .

[3] Julier, Jeffery K. Uhlmann, "A New Extension of the Kalman Filter to Nonlinear System," proceedings of American control conference, Seattle,WA, pp 1628-1632. 1995.

[4] E.A. Wan, R. Van der Merwe, "The Unscented Kalman Filter for nonlinear estimation," in Proceddings of IEEE symposium 2000 (AS-SPCC) Lake Louise, Canada pp. 153-158 October 2000.

[5] Oleksiy V.Korniyenko, Mohammad S.Sharawi, Oklahand University "Neural Network based approach for tuning kalman filter", 2005 IEEE International Conference on I.E.E.E. Electro/Information Technology Conference (EIT 2005), Lincoln - Nebraska, May 22-25, 2005
[6] Michail N. Petsios, Emmanouil G. Alivizatos, Nikolaos K. Uzunoglu, "Manoeuvring target tracking using multiple bistatic range and rangerate measurements", Science Direct, Signal Processing 87(2007) 665-686.

[7] Zhansheng Duan, X. Rong Li, Chongzhao Han, Hongyan Zhu, "Sequential Unscented Kalman Filter for Radar Target Tracking with Range Rate Measurements," $20057^{\text {th }}$ International Conference on Information Fusion (FUSION).

[8] R.G. Brown, P.Y.C. Hwang, "Introduction to Random Signals and applied Kalman Filtering," third ed., Prentice Hall1997.

[9] Goldberg, D.E., 1989. Genetic Algorithms in Search and Optimization. Addison-Wesley, Reading, MA.

[10] Dash, P.K., Panigrahi B.K., Shazia Hasan.: Hybrid Particle Swarm Optimization and Unscented Filtering Technique for Estimation of Nonstationary Signal Parameters. IETE journal of research, vol.55, issue 6, Nov-Dec (2009).

[11] K. Premalatha and A.M. Natarajan "Hybrid PSO and GA for Global aximization" Pg- 598-608 Int. J. Open Problems Compt. Math., Vol. 2, No. 4,December 2009.

[12] WANG MingHui, WAN Qun ,YOU ZhiSheng “A gate size estimation algorith $\mathrm{m}$ for data as sociation filters" Springer Journal on Information Sciences in China Series, Apr. 2008 vol. 51 no. 4 pg 425432

[13] Krzysztof Szabat and Teresa Orlowska Kowalska "Performance Improvement of Industrial Drives With Mechanical Elasticity Using Nonlinear Adaptive Kalman Filter" IEEE Transactions on industrial electronics, vol. 55, no. 3, march 2008

[14] H.A.P. Blom and Y. Bar-Shalom, "The Interacting Multiple Model Algorithms for Systems with Markovian Switching Coefficients", IEEE Trans. on Automatic Control, vol. 33(8), 1988.

[15] N.M. Kwok_, D.K. Liu, G. Dissanayak "Evolutionary computing based mobile robot localization" Elsevier Engineering Applications of Artificial Intelligence 19 (2006) 857-868

[16] Bernt M. A kesson, John Bagterp Jorgensen, Niels Kjølstad Poulsen, A generalized auto covariance least-squares method for Kalman filter tuning, $A$ journal of process control, Elsevier 2007

[17] Oleksiy V.Korniyenko, Mohammad S.Sharawi, Oklahand University ,'Neural Network based approach for tuning kalman filter", 2005 IEEE International Conference on Volume, Issue , 22-25 May 2005 Page(s): 1 - 5

[18] K.L Shee Y.K Wong "Speed Estimation of Induction motor drive using an optimized EKF" 
IEEE transaction on industrial electronics" Vol 49, No 1 February 2002 pp 124-133

[19]. Levent Ince a, Bulent Sezen b, Erhan Saridogan a, Huseyin Ince "An evolutionary computing approach for the target motion analysis (TMA) problem for underwater tracks Expert Systems with Applications 36 (2009) 3866-3879

[20] Kennedy, J. (1997), “The particle swarm: social adaptation of knowledge", Proceedings of IEEE International Conference on Evolutionary Computation, Indianapolis, IN, pp. 303-8.

[21] Dah-Jing Jwo, Shun-Chieh Chang, (2009), "Particle swarm optimization for GPS navigation Kalman filter adaptation", Aircraft Engineering and Aerospace Technology, Vol. 81 Iss: 4 pp. $343-$ 352

[22] SURENDRA RAO Scientist - E Naval Science and Technological Laboratory, Visakhapatnam "Artificial Neural Network Embedded Kalman Filter Bearing Only Passive Target Tracking" Proceedings of the 7th Mediterranean Conference on Control and Automation (MED99) Haifa, Israel - June 28-30, 1999

[23] B.J. Lee, J.B. Park, Y.H. Joo and S.H. Jin "Intelligent Kalman filter for tracking a manoeuvring target” IEE Proc.-Radar Sonar Navig., Vol. 151, No. 6, December 2004 pp 344350

[24] Zhi-Jun Yu, Shao-Long Dong Jian-Ming Wei, Tao Xing1, Hai-Tao Liu1 "Neural Network Aided Unscented Kalman Filter For Maneuvering Target Tracking In Distributed Acoustic Sensor Networks" Proceedings of the International Conference on Computing: Theory and Applications (ICCTA'07) (0-7695-2770-) 2007

\section{Authors' Profiles}

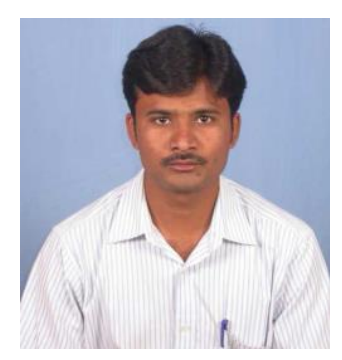

Ravi Kumar Jatoth was born in Warangal, India on October 13 1982. He received his B.E degree in Electronics and communications Engineering from Os mania University, Hyderabad in 2003; M.Tech in Instrumentation and Control Systems from Jawaharlal Nehru Technological University Hyderabad in 2005. He is currently working as Lecturer in National Institute of Technology-Warangal. His research areas include Digital Signal Processin, Tracking Algorithms and Embedded Systems. He has published papers in 8 international journals and 20 international conferences

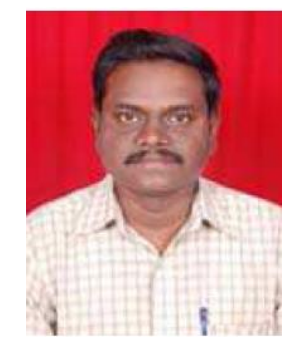

Dr.T.Kishore Kumar. (Member IETE,INDIA) received the B.Tech Degree in Electronics \& Communication Engineering and Master of Techonology in Digital Systems and Computer Electronics from Sri Venkateswara University and Jawaharlal Nehru Technological University India in the year 1992 and 1996 respectively and obtained Doctrate degree in Digital Signal Processing from Jawaharlal Nehru Technological University,Hyd India in 2004. From 1999 he was associated with NIT Warangal in the position of Associate Professor, prior to this he was selected for Cabinet Secretariat Prime Minister office Gov INDIA as Technical officer(Tele). His current areas of interest are signal processing and speech processing. At present he is guiding three Phd Scholars and two M.Tech Students. He has published several papers in the areas of Signal \& Speech Processing. He has participated in various courses such as Signal processing, VLSI and Curriculum development which are organized by reputed Institutions in India such as IIT Kharagpur, Intel Bangalore, IUCEE at Infosys, Mysore.

How to cite this paper: Ravi Kumar Jatoth, T. Kishore Kumar,"Performance Comparison of Hybrid GA-PSO Based Tuned IMMs for Maneuver Target Tracking", International Journal of Intelligent Systems and Applications(IJISA), vol.5, no.12, pp.120-134, 2013. DOI: 10.5815/ijisa.2013.12.11 